\title{
E-Cigarette Survey among Student Pharmacists Reveals Troubling Results
}

\author{
Jamie L. McConaha1, Phillip Lunney², Jan M. Kitzen ${ }^{3}$ Joseph V. Pergolizzi Jr.4, Robert Taylor Jr. ${ }^{4}$, \\ Robert B. Raffa ${ }^{5,6,7}$
}

${ }^{1}$ Duquesne University School of Pharmacy, Pittsburgh, PA, USA

${ }^{2}$ Lachman Consultants, Westbury, NY, USA

${ }^{3}$ Kitzen Pharmaceutical Consulting, Collegeville, PA, USA

${ }^{4}$ NEMA Research, Naples, FL, USA

${ }^{5}$ University of Arizona College of Pharmacy, Tucson, AZ, USA

${ }^{6}$ Neumentum, Inc., Palo Alto, CA, USA

${ }^{7}$ Temple University School of Pharmacy, Philadelphia, PA, USA

Email: jkitzen@verizon.net

How to cite this paper: McConaha, J.L. Lunney, P., Kitzen, J.M., Pergolizzi Jr., J.V., Taylor Jr., R. and Raffa, R.B. (2020) E-Cigarette Survey among Student Pharmacists Reveals Troubling Results. Pharmacology \& Pharmacy, 11, 127-136. https://doi.org/10.4236/pp.2020.116012

Received: May 18, 2020

Accepted: June 27, 2020

Published: June 30, 2020

Copyright ( 2020 by author(s) and Scientific Research Publishing Inc. This work is licensed under the Creative Commons Attribution International License (CC BY 4.0).

http://creativecommons.org/licenses/by/4.0/

\begin{abstract}
The rise in "vaping"-associated deaths in the United States raises serious concerns. A justification for some level of risk is that e-cigarettes might provide a "reverse-gateway" from smoking traditional cigarettes to a less-harmful alternative. But are users actually smokers? We developed an electronic on-line survey to gather data regarding e-cigarette usage in a target study population of pharmacy students. The survey was created using Google Forms to collect the responses anonymously. We surveyed medical-savvy healthcare students about their e-cigarette use, and whether or not their healthcare providers were aware of their e-cigarette use. Although nearly one-fourth of a convenience sampling of 134 pharmacy-student respondents (23.9\%) reported using e-cigarettes, only $2.0 \%$ reported prior regular cigarette use, $28 \%$ used cartridges containing nicotine and only $11.2 \%$ had tried and wanted to quit. The majority (64.1\%) reported that their healthcare providers did not ask about such use, and respondents did not volunteer this information. The results of this pilot survey reveal a significant e-cigarette use among health-aware pharmacy students, and they do not support the notion that the respondents do so to quit smoking. The observation that most of the students' healthcare providers did not inquire about their e-cigarette use, coupled with the finding that users did not volunteer their vaping behavior, suggests that the information should be included when getting a medical history. Further research is needed to determine what behavioral factors may play a role in this type of decision-making among student healthcare professionals.
\end{abstract}




\section{Keywords}

E-Cigarettes, Nicotine, Smoking Cessation, Survey, Vaping

\section{Introduction}

Since 1964, the United States Surgeon General has released at least 30 reports related to the morbidity and mortality attributed to smoking and secondhand smoke. The harmful effects of these products are well known, yet the statistics surrounding the numbers of persons adversely affected by their use remain high. Cigarettes are still responsible for one in five deaths in the United States, and the chronic diseases caused by tobacco use are leading causes of death and disability. The economic burden of cigarette use in the U.S. includes more than $\$ 193$ billion annually in healthcare costs and loss of productivity [1].

Despite these sobering statistics, the good news is that years of public health efforts have proven successful in reducing cigarette usage from their historical highs. Cigarette smoking has fallen to its lowest point in recorded history, according to new data from the Centers for Disease Control and Prevention (CDC). In 2016, an estimated 15.5\% (37.8 million) of U.S. adults were current cigarette smokers. In 2017, this number dropped to 14\% [2] [3]. Strategies such as the rising cost of tobacco products, consumer education, and assistance in cessation efforts are the primary drivers behind the decline in cigarette use. Another major reason may be the increasing prevalence of electronic cigarette (e-cigarette) use.

Electronic nicotine delivery systems (ENDS) are battery powered devices that provide doses of nicotine and/or other additives in the form of an aerosol. Introduced into the United States in 2007, there are currently numerous types of ENDS devices available, with e-cigarettes being the most common. It was an early fundamental argument that smoking e-cigarettes is preferable health-wise to smoking traditional cigarettes, and that the majority of e-cigarette users are current smokers of traditional cigarettes [4] [5]. And there is some evidence that some users of e-cigarettes indeed do use them as a smoking cessation aid, despite their lack of approval and good evidence of effectiveness for this purpose. Concurrently, there has been a rapid surge in the numbers of users of e-cigarettes, particularly concerning by younger adults and persons with medical conditions [6]. Anecdotal evidence suggests that many teens are now "experimenting" with e-cigarette devices. Preliminary federal data show a $78 \%$ surge in high school students using e-cigarettes, prompting the FDA to label this increased use, together with highly-publicized deaths, as an "epidemic" [7].

\section{Background and Need}

The use of e-cigarettes ("vaping") by young persons is a particularly troubling emerging public health concern. Among high school students, the use of 
e-cigarettes now exceeds the smoking of conventional cigarettes [8]. The popularity of e-cigarettes, particularly their use in the young, is of concern for health professionals in part because of the common misconception that these products are harmless and that they are nicotine-free. While the long-term health effects of e-cigarettes are still unknown, one concern is that these products, counter to the benevolent goal, in reality act as a "gateway drug" to cigarette smoking or, in the extreme, illicit drug use.

Many e-cigarette products contain nicotine, some in concentrations greater than in cigarettes. Not only is nicotine addictive, but it impairs brain and lung development if used during adolescence [9]. Brain imaging studies of adolescents who began smoking at a young age had markedly reduced activity in the prefrontal cortex of the brain, an area critical for a person's cognitive behavior and decision-making. This can lead to increased sensitivity to other drugs and greater impulsivity [9].

A specific brand of e-cigarette (Juul) has become extremely popular among children and adolescents. Launched in 2015, it currently holds more than $70 \%$ of the U.S. market [7]. The nicotine content found in "Juuls" is also of concern. The e-liquid in one Juul is $5 \%$ nicotine by volume, which is more than double the concentration of nicotine present in other e-cigarettes. Teens often use multiple Juul pods in one sitting, exposing them to a risk of toxicity and greatly increasing their risk of nicotine addiction. Whereas, some of the other e-cigarette brands contain a chemically-modified form of nicotine called "freebase nicotine," Juul contains "nicotine salts", which are more readily (rapidly) absorbed into the bloodstream.

Perhaps what is most striking is that some users of Juuls do not consider them to be an e-cigarette. The Yale Tobacco Center for Regulatory Science reports that when teens are asked about their e-cigarette habits, they do not consider the use of Juuls ("juuling”) to be part of that activity [10].

\section{Target Population}

Pharmacists are highly regarded by the public as trusted healthcare professionals and as a source of information about contemporary issues related to healthcare. As some of the most accessible healthcare providers (HCP), pharmacists play an integral role within the healthcare system. The role of the pharmacist in providing patient counseling and smoking cessation services in particular is well documented. Unfortunately, due to the paucity of research in this area, many pharmacists are untrained how to counsel (potential) users of e-cigarettes.

The Accreditation Council for Pharmacy Education (ACPE) provides professional standards that schools of pharmacy must meet. These standards include educational outcomes on topics such as foundational knowledge, practice and care, and professional development. In addition to their didactic education, student pharmacists also must participate in required pharmacy practice experiences. Pharmacy sites used for these practice experiences need to demonstrate 
a commitment to health promotion and disease prevention, of which smoking-cessation counseling is included as an activity for students to meet this requirement. Given the required inclusion of smoking-cessation in the pharmacy school curriculum along with practical experiences in counseling patients on smoking cessation, student pharmacists are well versed about the dangers of cigarette smoking. Therefore, anecdotal stories, including observations from pharmacy school professors, that many student pharmacists use e-cigarettes would seem to reasonably suggest that they do so in order to break the habit of smoking cigarettes. But is this really the case? This study targeted student pharmacists in the professional curriculum (last four years of pharmacy school) to gather data on the extent and intent of their usage of e-cigarettes.

\section{Materials and Methods}

We developed an electronic on-line survey to gather data regarding e-cigarette usage in the target study population. The survey was created using Google Forms, which allowed collection of the responses anonymously. The survey and research study were approved by Duquesne University Institutional Review Board (IRB). E-mail blasts were sent to all of the Duquesne University School of Pharmacy students in the professional phase of the curriculum (PY1-PY4). The survey was ( ${ }^{*}$ denotes a required response):

1. Age $^{*}$

2. Do you currently reside in the United States?*

○ Yes

○ No

3. What is your zip code of residence?*

4. Are you currently in school or employed?*

- Employed full-time

O Full-time student

○ Student but work part-time

- Not currently employed or in school

O Other

5. Height*

6. Weight (lbs)*

7. Self-identify*

○ Male

Female

O Other

8. Who lives in your household? Please check all that apply*
○ P Parents
$\bigcirc$ __ Siblings
○ C Children
○ _ Roommates
○ __ Other 
9. Do you have any underlying medical conditions? If yes, please describe

10. Do you regularly smoke?*

○ Yes

○ No

If yes, how many cigarettes do you smoke a day (average)?

11. Do you currently use other forms of tobacco?*

O Yes

○ No

If yes, what other forms of tobacco?

12. If using a form of tobacco other than a cigarette, how often/how much do you use?

13. Do you currently use e-cigarettes (vaping)?*

O Yes

○ No

If yes, how often do you use?

14. What led you to first try an e-cigarette? Select all that apply*

○ _ Flavors

$\bigcirc$ __ Smoking cessation aid

○ __ Weight loss

○ __ Looks cool

$\bigcirc$ _ Peer usages

○ _ Curiosity

O __ Use by family or friends

○_ Cost less vs cigarettes and other forms of tobacco

○ __ Other

15. What type of e-cigarette do you use?*

○ _ "ciga-like" (ex: Blu, Juul, etc.)

- "e-Gos"

○ "Mods"

○ _ Unsure

$0 \ldots$ I I do not use e-cigarettes

16. What level nicotine cartridge do you use with your e-cigarettes?*

○ Low

○ Medium

O High

○ No nicotine

17. Have you ever attempted to quit using e-cigarettes?*

○ Yes

○ No

18. How many e-cigarette quit attempts have you experienced?*

19. Has a healthcare professional (physician, dentist, pharmacist, etc.) ever asked you about e-cigarette use?*

○ Yes

○ No 
20. Have you ever disclosed your use of e-cigarettes to a healthcare professional?*

○ Yes

○ No

If yes, were you provided any counseling or information regarding their use?*

○ Yes

○ No

○ N/A

21. Have you ever hidden your use of e-cigarettes?*

○ Yes

○ No

22. Have you ever participated in a focus group?*

○ Yes

○ No

O Maybe

○ N/A

If no, would you be interested in participating in a focus group?*

○ Yes

○ No

O Maybe

○ N/A

23. If you answered yes to participating in a focus group, please enter your e-mail address

In the following questions, please indicate whether you agree or disagree with each statement. $(0=$ completely disagree, $10=$ completely agree $)$

24. Electronic cigarettes are safe

$\begin{array}{lllllllllll}0 & 1 & 2 & 3 & 4 & 5 & 6 & 7 & 8 & 9 & 10\end{array}$

25. E-cigarettes do not produce second-hand smoke

$$
\begin{array}{lllllllllll}
0 & 1 & 2 & 3 & 4 & 5 & 6 & 7 & 8 & 9 & 10
\end{array}
$$

26. I can stop using e-cigarettes any time that I want

$$
\begin{array}{lllllllllll}
0 & 1 & 2 & 3 & 4 & 5 & 6 & 7 & 8 & 9 & 10
\end{array}
$$

27. I plan to continue using e-cigarettes for the foreseeable future

$$
\begin{array}{lllllllllll}
0 & 1 & 2 & 3 & 4 & 5 & 6 & 7 & 8 & 9 & 10
\end{array}
$$

Two e-mail blasts were sent one month apart. Completion of the survey was voluntary, and no incentive was received for responding. Inclusion criteria for participation in the study included any student pharmacist between the ages of 18 and 24 years. There were no exclusion criteria. All information was collected from the Google Forms survey and exported into an Excel ${ }^{\circ}$ worksheet.

\section{Results}

The original survey data contained the results obtained from more than 200 participants which were subjected to Exploratory Data Analysis. The analysis sug- 
gested that the data represented two populations with respect to age, including the target population of full-time pharmacy students $(n=134)$, and an older group working at full- or part-time jobs. Only the survey responses for the students were analyzed for this study.

\subsection{Demographic Summary}

The only demographic questions included in the survey were age, gender self-identity, height and weight. The average age of the 134 students was 22.5 years and ranged from a minimum of 17 to a maximum of 39 . The sample included $81.3 \%$ females $(n=109)$ and $18.7 \%(n=25)$ male respondents. The height and weight measurements were converted to BMI indices to remove gender differences. The average BMI for the sample was 24.5. Individual values were converted to ordinal scale classification based on NIH guidelines. Based on this classification, $53.0 \%(\mathrm{n}=71)$ of the respondents were Normal or Underweight (BMI $\leq 24.9)$, whereas $31.3 \%(\mathrm{n}=42)$ were overweight (BMI 25 - 29.9), and $15.7 \%(\mathrm{n}=21)$ were obese $(\mathrm{BMI}>30)$.

\subsection{Questions Related to Smoking and E-Cigarette Use}

The results for questions concerning the use of tobacco and e-cigarettes are presented in Table 1 . Only $2 \%$ of respondents confirmed that they were regular smokers and only $3.7 \%$ confirmed that they used other forms of tobacco, indicating a very low incidence of tobacco use as compared to the general population. In contrast, $23.9 \%$ confirmed that they currently vaped e-cigarettes. The order of magnitude increase observed for vaping versus traditional tobacco products in this population suggests that the products are being used recreationally and not as an aide for smoking cessation. In related questions, $11.2 \%(\mathrm{n}=$ 16) of the respondents reported that they had attempted to quit vaping, and $28 \%$ $(n=37)$ confirmed that they used e-cigarette products containing nicotine.

The bottom row of Table 1 represents the responses collected for determining the role of Health Care Providers with vaping. The majority of respondents (64.1\%) had never been asked by an HCP (physician, dentist, or pharmacist) about e-cigarette use.

\subsection{Perceptions about E-Cigarette Safety Based on a Rating Scale}

Four questions on the survey (questions 24 - 27) were included in order to measure the perceived safety of e-cigarettes using a Likert scale ( 0 to 10 , with 0 indicating that the respondent completely disagrees and 10 indicating complete agreement with the statement). For this scale a response of 5 would indicate neutrality. The summary results for these questions are shown in Table 2.

In all cases, the mean was less than 5, suggesting that the respondents disagreed on average with the statements. The strongest disagreement was recorded for the statement "I plan to continue using e-cigarettes for the foreseeable future" (mean $=1.35)$. The coefficient of variation was high $(>100 \%)$ for all statements with the exception of "I can stop using e-cigarettes any time I want" 
Table 1. Responses to questions related to smoking and e-cigarette use.

\begin{tabular}{|c|c|c|}
\hline Question & $\begin{array}{c}\text { Answer }=\text { NO \% } \\
{[\mathrm{n}]}\end{array}$ & $\begin{array}{c}\text { Answer }=\text { YES \% } \\
{[n]}\end{array}$ \\
\hline Do you regularly smoke? & $98.0[131]$ & $2.0[3]$ \\
\hline Do you currently use other forms of tobacco? & $96.3[129]$ & $3.7[5]$ \\
\hline Do you currently use e-cigarettes (vaping)? & $76.1[102]$ & $23.9[32]$ \\
\hline $\begin{array}{l}\text { Has a healthcare provider ever asked you about } \\
\text { e-cigarette use? }\end{array}$ & $64.1[86]$ & $35.9[48]$ \\
\hline
\end{tabular}

Table 2. Summary statistics for perceptions concerning e-cigarette safety, as measured on a rating scale.

\begin{tabular}{lcccccc}
\hline \multicolumn{1}{c}{ Question } & Mean & $\begin{array}{c}\text { Std } \\
\text { Dev }\end{array}$ & $\begin{array}{c}\text { COV } \\
\%\end{array}$ & $\begin{array}{c}\text { Mean } \\
\text { L95\% }\end{array}$ & $\begin{array}{c}\text { Mean } \\
\text { U95\% }\end{array}$ & N \\
\hline e-Cigs are safe & 2.02 & 2.30 & 113.87 & 1.63 & 2.42 & 132 \\
e-Cigs do not produce 2 $2^{\text {nd }}$-hand smoke & 3.28 & 3.17 & 96.56 & 2.74 & 3.82 & 133 \\
I can stop using e-Cigs any time I want & 4.04 & 0.36 & 8.91 & 5.36 & 6.78 & 126 \\
I plan to use e-Cigs for the fore-seeable future & 1.35 & 2.71 & 201.20 & 0.87 & 1.82 & 127 \\
\hline
\end{tabular}

Abbreviations: Std Dev = standard deviation of the mean; COV = coefficient of variation; L95\% = lower 95\%; U95\% = upper 95\%.

$(\mathrm{COV}=8.91 \%)$. This suggests that some of the variation in the responses was due to an independent factor. Thus, the hypothesis was tested that the variation in the results was due to the single demographic factor "Gender Identity". This hypothesis was tested using the random two-group comparison (analysis of variance) in JMP software version 11 (SAS Institute). Statistical significance was assessed at the $95 \%$ confidence level.

In all four cases, a statistically significant difference in perception was observed for females versus males at the $95 \%$ confidence level or higher (data not shown). For each of these four questions, females provided lower mean responses than males, indicating that they disagreed more strongly with the statements. This suggests that males are more likely than females to ignore or discount the risks of vaping.

\section{Discussion}

The results of our survey are surprising and disconcerting, and may offer insight into the recent increase in vaping [11] that has resulted in serious adverse events and deaths [12] [13]. In this sample of medically-savvy future healthcare professionals (pharmacy students) there was a high prevalence of vaping behavior (23.9\%). Perhaps the most disappointing result is that the population we studied was not using e-cigarettes as a smoking cessation attempt, indeed almost none of the respondents (only 2.0\%) were regular smokers of conventional cigarettes and almost all (96.3\%) were not using any form of tobacco at all. Counter intuitively, although most of the respondents tended to disagree with 
the statement "e-cigarettes are safe", nearly one in four of the respondents $(23.9 \%)$ stated that they were currently vaping. This suggests that, despite the fact that they were aware of the unsafe nature of vaping, they were willing to indulge in risky behavior. An explanation for this disconnect was not elicited in the present pilot survey, but should be of major concern, and the subject of subsequent inquiry. In this regard, it is perhaps of note that the gender-identity analysis suggests that males are significantly more likely than are females to engage in vaping.

A rather eye-opening observation to emerge from the survey results was the lack of involvement of HCPs in asking about the vaping behavior of their patients. Less than $36 \%$ of the respondents indicated that their HCP ever asked about vaping use. Whether or not this indicates a lack of safety concern on the part of the HCP or is due to some other factor is difficult to tell from our results. Nevertheless, especially when considering recent cases of vaping related lung injury [12] [13] (which became publicized after our survey was completed) this question should now be considered to be a routine health background query by all HCPs in a manner similar to their concern about lifestyle activities such as diet and exercise, smoking conventional cigarettes, or alcohol use.

\section{Conclusion}

In conclusion, the results of our survey suggest that a significant number of pharmacy students are using e-cigarettes; almost none of them are using e-cigarettes to taper off traditional cigarettes; the use persists despite the knowledge that it could be risky; and there seems to be a culture of "don't ask, don't tell" with their HCPs. Therefore, there is a pressing need to increase the awareness of healthcare students, as future providers of health information to the public, about the risks that are associated with vaping the currently-formulated (or worse, adulterated) products for recreational purposes. This should not, though, rule out an awareness of the possible favorable relative benefit-risk for some people of using e-cigarettes as a risk-mitigation strategy or assist to taper off traditional cigarette use.

\section{Conflicts of Interest}

The authors declare no conflicts of interest regarding the publication of this paper.

\section{References}

[1] Surgeon General (2010) How Tobacco Smoke Causes Disease: The Biology and Behavioral Basis for Smoking-Attributable Disease: A Report of the Surgeon General. U.S. Department of Health and Human Services, Atlanta, GA. https://www.ncbi.nlm.nih.gov/books/NBK53017/

[2] Centers for Disease Control and Prevention (2019) Burden of Tobacco Use in the U.S.: Current Cigarette Smoking among U.S. Adults Aged 18 Years and Older. CDC. 
https://www.cdc.gov/tobacco/campaign/tips/resources/data/cigarette-smoking-in-u nited-states.html\#three

[3] Jamal, A.P.E., Gentzke, A.S., Homa, D.M., Babb, S.D., King, B.A. and Nef, L.J. (2019) Current Cigarette Smoking among Adults: United States, 2016. MMWR Morb Mortal Wkly Report. https://doi.org/10.15585/mmwr.mm6702a1

[4] McMillen, R.C., Gottlieb, M.A., Shaefer, R.M., Winickoff, J.P. and Klein, J.D. (2015) Trends in Electronic Cigarette Use among U.S. Adults: Use Is Increasing in Both Smokers and Nonsmokers. Nicotine \& Tobacco Research, 17, 1195-1202. https://doi.org/10.1093/ntr/ntu213

[5] Delnevo, C.D., Steinberg, M.B., Villanti, A.C., Pearson, J.L., Niaura, R.S. and Abrams, D.B. (2016) Patterns of Electronic Cigarette Use among Adults in the United States. Nicotine \& Tobacco Research, 18, 715-719. https://doi.org/10.1093/ntr/ntv237

[6] Mirbolouk, M., Charkhchi, P., Kianoush, S., Uddin, S.M.I., Orimolove, O.A., Jaber, R., Bhatnagar, A., Benjamin, E.J., Hall, M.E., DeFilippis, A.P., Maziak, W., Nasir, K. and Blaha, M.J. (2018) Prevalence and Distribution of E-Cigarette Use among U.S. Adults: Behavioral Risk Factor Surveillance System, 2016. Annals of Internal Medicine, 169, 429-438. https://doi.org/10.7326/M17-3440

[7] Anonymous (2018) FDA Moves forward with E-Cigarette Curbs to Reduce Teen Vaping. https://fortune.com/2018/11/15/fda-e-cigarette-measures-underage-vaping/

[8] U.S. Department of Health and Human Services (2016) E-Cigarette Use among Youth and Young Adults. A Report of the Surgeon General. National Center for Chronic Disease Prevention and Health Promotion, Office on Smoking and Health, Atlanta, GA.

https://e-cigarettes.surgeongeneral.gov/documents/2016 sgr full report non-508.p df

[9] Fraga, J. (2019) The Dangers of Juuling. http://www.center4research.org/the-dangers-of-juuling/

[10] Raven, K. (2018) Your Teen Is Underestimating the Health Risks of Vaping. Yale School of Medicine, Yale Tobacco Center of Regulatory Science. https://medicine.yale.edu/psychiatry/tobacco/news-article/18263/

[11] Hswen, Y. and Brownstein, J.S. (2019) Real-Time Digital Surveillance of Vaping-Induced Pulmonary Disease. The New England Journal of Medicine, 381, 1778-1780. https://www.nejm.org/doi/full/10.1056/NEJMc1912818 https://doi.org/10.1056/NEJMc1912818

[12] Lewis, N., McCaffrey, K., Sage, K., Green, J., Goldstein, L., Campbell, H. and Ferrell, D. (2019) E-Cigarette Use, or Vaping, Practices and Characteristics among Persons with Associated Lung Injury: Utah, April-October. The Morbidity and Mortality Weekly Report, 68, 953-956. https://doi.org/10.15585/mmwr.mm6842e1

[13] Blount, B., Karwowski, M.P., Morel-Espinosa, M., Rees, J., Sosnoff, C., Cowan, E. and Gardner, M. (2019) Evaluation of Bronchoalveolar Lavage Fluid from Patients in an Outbreak of E-Cigarette, or Vaping, Pproduct Use-Associated Lung Injury: 10 States, August-October. The Morbidity and Mortality Weekly Report, 68, 1040-1041. https://www.cdc.gov/mmwr/volumes/68/wr/mm6845e2.htm?s cid=mm6845e $2 \mathrm{w}$ https://doi.org/10.15585/mmwr.mm6845e2 